\title{
PRECISION IMPROVEMENT AND DELAY REDUCTION IN SURGICAL TELEROBOTICS
}

\author{
Dr. Samuel Manoharan, \\ Professor, Department of Electronics, \\ Bharathiyar College Of Engineering and Technology, \\ Thiruvettakudy, Karaikal, India. \\ Email id: jsamuel@,bcetedu.in \\ Dr. Narain Ponraj, \\ Assistant Professor, \\ Karunya University, \\ Coimbatore, India. \\ Email id: narainpons@karunya.edu
}

\begin{abstract}
The field of medicine is greatly benefited by telerobotic surgeries. It allows the surgeon to reach the patient remotely. Traditional surgical telerobotic techniques had several drawbacks mainly due to communication delay and lack of precision. Signal latency also causes significant delay in operations. This also causes the risk of increased human errors. The surgical workflow can be altered by the addition of features like self-correction and self-automation. In this paper, we reduce the delay in telerobotic surgery using supervisory control approach and apply a haptic feedback to regulate safety. Motion scaling technique is used to combat responses in delayed environment as well as to improve the accuracy of the system. Augmented reality, improved functional design, comfort and skills along with the ability to reach the patient remotely promotes the wide use of surgical telerobotics.
\end{abstract}

Keywords: Telerobotics, surgical instruments, motion scaling, haptic feedback, supervisory control

\section{INTRODUCTION}

The advances in medical science and technology has enabled maximizing patients experience with the inclusion of interventions, novel devices, interfaces to network with data. Telemedicine virtually brings professionals to regions where medical amenities and specialists are not available. It is possible to perform tasks from a remote and safe location with the help of telerobotics and teleoperation. This system reduces inconveniences and cost and improves information access. In this system, a human operator transmits control signals in the form of commands to a robot that performs the task in a remote environment. The feedback from the robot (slave) is returned to the operator (master) from cameras, haptic devices and such sensors in case of bilateral teleoperation. In case of unilateral telesurgery, there is no force feedback provided to the user. 
Journal of Artificial Intelligence and Capsule Networks (2019)

Vol.01/ No. 01,

Pages: 28-36

http://irojournals.com/aicn/

DOI: https://doi.org/10.36548/jaicn.2019.1.004

The force feedback and displays gives a virtual presence feeling to the operator. The first telesurgery was performed in 2001, termed as 'Charles Lindbergh operation'. It was a 45 minute laparoscopic cholecystectomy [10] implemented by ZEUS robot, where the surgeon was located in New York and the patient in France. Other than this transatlantic robot assisted surgery, Da Vinci robotic system and video assisted surgery are notable telesurgery systems [14]. The surgical robots depend on the controls that acts as an interface between the robots and the surgeons that converts the movement of the surgeon into robotic commands [13]. Fundamentally, there is a huge variance among several aspects associated with the use of body and hands as surgical gears in the operating room.

Telepresence is the fundamental framework of telerobotics. The data regarding remote environment is conferred to the operator in a natural manner. This creates the feeling of physical presence in the remote location. Telecommunication network institutes the master-slave system. Medical robots are developed so as to provide accuracy, steady-hand motion, bio-motion compensation, motion scaling and various other critical features. In early telesurgeries, remote operation was carried out using inputs from tools like keyboard, monitor, joystick and other peripherals.

Telesurgery is developing at a fast phase with the advancements in robotics and telecommunication engineering. For complete adoption and clinical integration of telesurgery, several factors such as financial and legal concerns, safety, economy and ethical concerns are to be taken care of [4]. Cyber-attacks, failure in establishing connection between master and slave sites, licensure applicability barrier and so on are major threats and concerns for telesurgery. Time delay and latency is a notable concern in telesurgery. It results in increasing the duration of operation and is prone to cause surgical inaccuracy. This can delay the patient recovery and risk safety of the patient.

\section{EXISTING LITERATURE}

A telesugery method based on eye-tracking in order to produce haptic restrictions with 6 degrees of freedom was proposed by Myloans et al [1]. The distance between the fixation point of the eyes, the fundamental force profile and the surgical apparatus relates with the force opposed to the surgeon's movement. This can circumvent unnecessary activities of the robot. Robert et al. [6] proposed a teleoperational mobile robot called TeMoto that uses robot operating system. This system enables community access, extensibility and hardware agnosticism. The paper presents metrics like operator context switching and task completion efficiency along with standard features like completion time, user studies, number of steps and so on for evaluation of user interface. 
Journal of Artificial Intelligence and Capsule Networks (2019)

Vol.01/ No. 01,

Pages: 28-36

http://irojournals.com/aicn/

DOI: https://doi.org/10.36548/jaicn.2019.1.004

André et al. [5] performed a systematic study of the research systems on touch-less human-computer interaction in surgery and radiology suites. Jasmeet et al. [7] proposed an approach to control the surgical robot's force despite the signal delay with the help of model predictive control (MPC) scheme. This scheme compensates deterministic time deferments and develops haptic feedback. Chao Liu et al. [3] proposed an efficient teleoperation system that provided a transparent and stable performance that offered robustness against environmental parameter variations and delay in communication. The paper presented a system based on nonlinear Hunt-Crossley model and used recursive least square (RLS) technique.

Thomas et al. [8] reviewed on research based on time delay in space teleoperation. Free position is done with the help of predictive display. The paper proposes plausible solutions for space programs while involving in telesurgery. In order to accomplish best competence, mixing procedures is suggested as a growing consensus. Reliability, cost and bandwidth considerations are also taken into account for optimal performance. Soheil et al. [9] presented a teleoperator that provides ideal performance and uses smith predictor and amplified wavevariables for reducing the effects of time-delay.

Hao $\mathrm{Su}$ et al. [15] presented a telesurgery system that is magnetic resonance image guided and uses pneumatic haptic device as a master robot and Fabry-Perot interferometry force sensor. This telesurgery offered a noise reduction ratio of less than $17 \%$ during simulation. Figure 1 represents the tracking controls of accuracy in position, speed and error. The motion of the master robot is tracked by the slave for a duration on $85 \mathrm{~s}$ with an RMS error of $0.11 \mathrm{~mm}$.
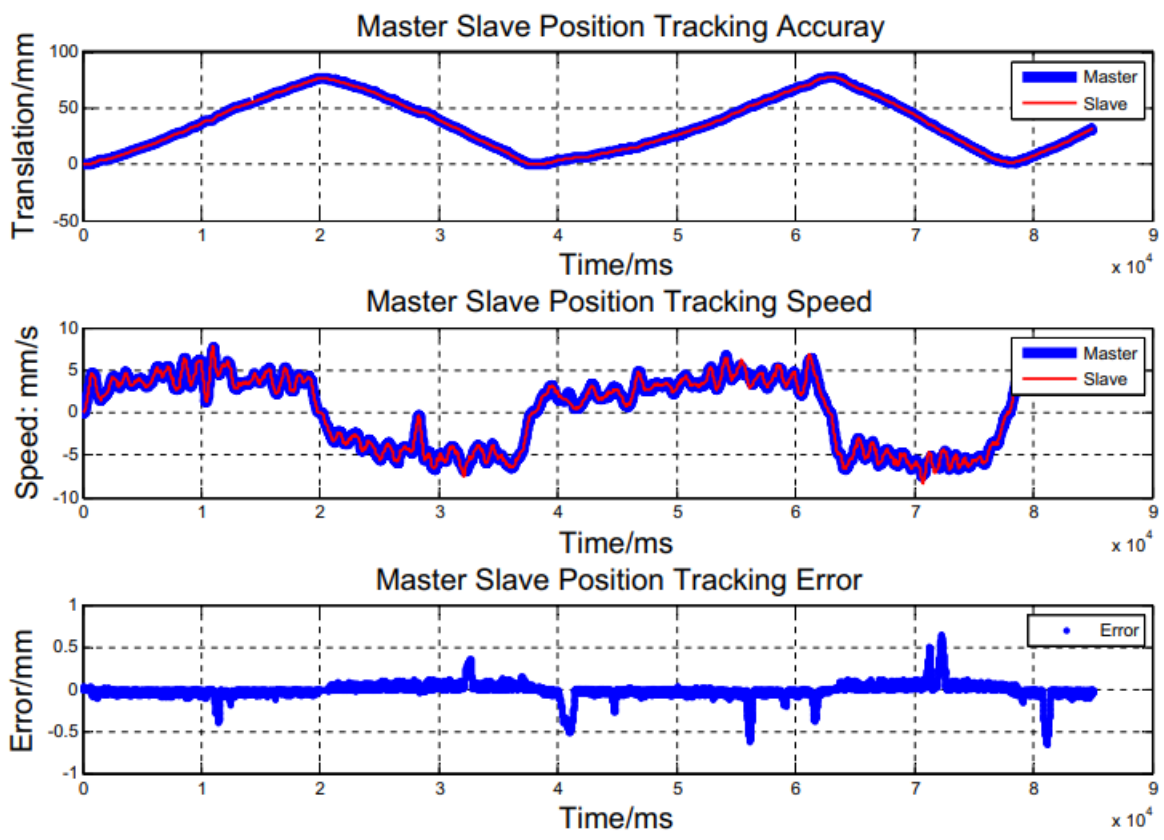
Figure 1 Tracking results of master-slave position, speed and error [15]

Qi Zhang et al. [16] proposed a tactile robotic telesurgery enabled with 5G technology. It addresses certain concerns like packet loss, jitter and latency that occurs with haptic feedback. The proposed technology meets the Quality of Service requirements with the help of $5 \mathrm{G}$ communication system. It provides ultra-low latency and mobile edge computing with network slicing and artificial intelligence. Mark Reichenbach et al. [17] performed research on miniature robots for telesurgery in scattered expedition environment to leverage surgical proficiency. The authors conducted an experiment for testing the capability of the miniature surgical robots with off-the shelf wireless equipment.

\section{PROPOSED WORK}

Figure 2 represents a teleoperation system flow chart where $\mathrm{d}$ represents the round trip delay and $\mathrm{m}$ represents the position of the master. The position of the target slave from the master is given by $\mathrm{s}_{\mathrm{m}}$, slave position is given by $s$ and the feedback to operator is represented by $I$. They system sampling rate is given by $f_{s}$ and $\mathrm{n}_{\mathrm{d}}=\mathrm{f}_{\mathrm{s}} \mathrm{d}$.

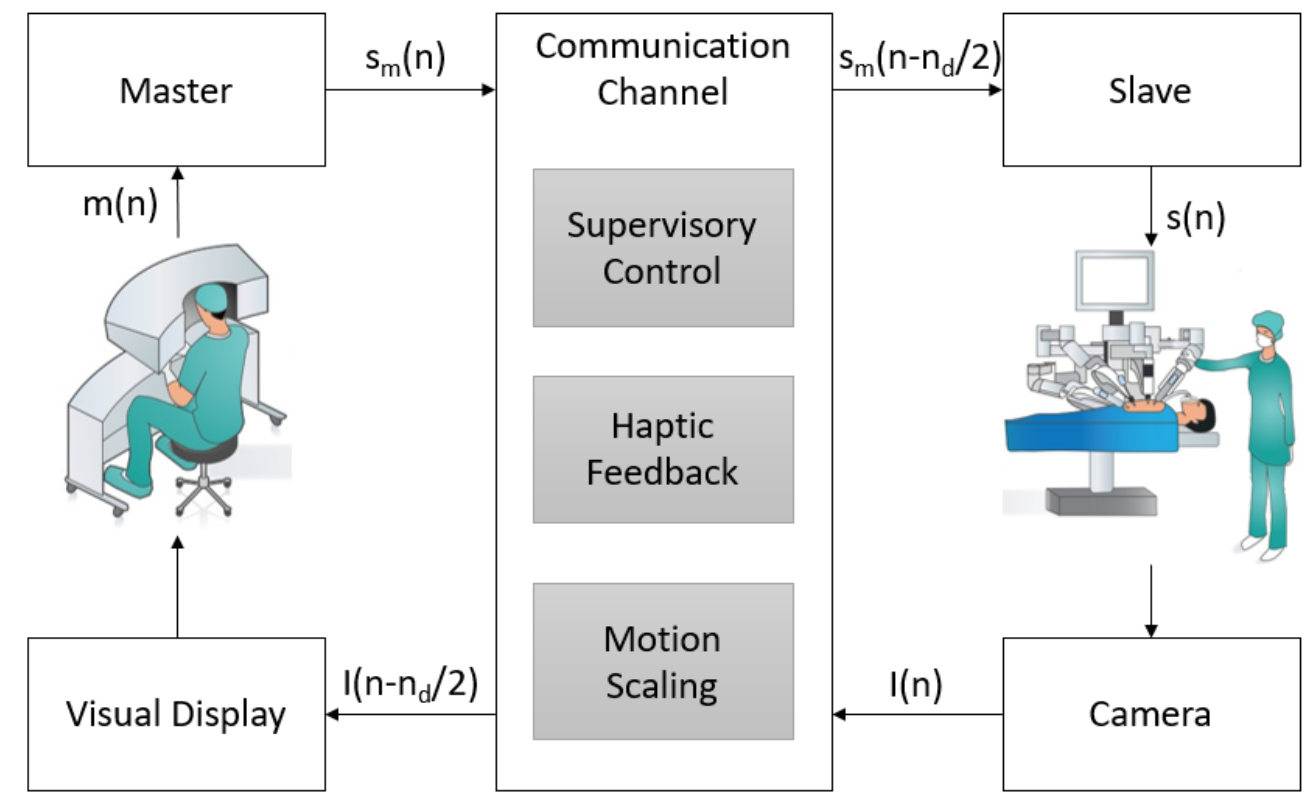

Figure 2 Teleoperation system with enhanced communication channel

\section{Supervisory Control}


Journal of Artificial Intelligence and Capsule Networks (2019)

Vol.01/ No. 01,

Pages: 28-36

http://irojournals.com/aicn/

DOI: https://doi.org/10.36548/jaicn.2019.1.004

For monitoring the input commands continuously, a structured supervisory control called HumanRobot Interaction (HRI) is used. The input commands include voice commands, hand pose and scaling factor. The graphical guidance and related constraints are processed by the visualization manager (VM). Based on the state of the HRI agent inferred by the VM, instinctive visual feedback is generated. With this system, the operator can recognize motion constraints with the help of voice commands. Based on the Levels of Autonomy (LOA) [11], the semiautonomous proficiencies of a robot and its spectrum is defined.

The low level commands for the operation of wheels, joints, grippers and so on are located at the lower end of the spectrum. These commands and control are tedious, time consuming and prone to error but are essential for operation. Time delay between transfer of commands and reception of feedback is a lingering issue with this type of control. This delay affects the success rate and productivity. Supervisory control can help overcoming issues of excessive cognitive load and transmission delay.

High level instructions are accepted by supervisory control. Integrated subtasks like grasping, motion planning and collision avoidance are executed by the remote system as one distinct action to the operator. Supervisory controllers are exposed to dynamic environmental changes that disturb semi-autonomous behaviour of the robot. By increasing the LOA of the system, these issues can be overcome.

\section{Haptic Feedback Technology}

Tactile information is transmitted to the teleoperator with the help of Haptic Feedback Technology [2]. The surgeon can feel the texture of the tissue during surgery through haptic feedback. This feedback is critical as it allows the surgeon to adjust the pressure applied on the instruments according to the tissue's consistency. While performing acute operations tying knots, dissection of tissue planes and so on, it is possible to cause damage to tissues if excess of tension is created. Similarly, in case the pressure applied is insufficient, it can result in suboptimal outcome. Earlier, telesurgery depended exclusively on visual feedback [12].

\section{Motion Scaling}

Motion scaling advances the performance of the teleoperation and reduces errors with minimum changes. It helps overcoming delay, error reduction, improvement of patient safety, develop intuitive control, simplify implementation and enable easy deployment of the system. Constant scaling factor can be lowered in order to improve the accuracy and time required for completion of task. Velocity scaling is used for improving the accuracy and reducing the cost of time. This type of scaling was built on the impression that an operator will 
travel slow when high precision is necessary. Positional scaling decreases scaling as the slave arms transport towards an obstacle.

\section{RESULT}

Before field implementation, it is essential to evaluate the performance of the system with simulation techniques. The factors to be considered during evaluation includes the success rate or the rate of completion of the task, the efficiency that includes steps involved, accuracy and so on, time delay and duration of completion of the task and the reduction of training time, cognitive load etc.

Task completion rate is validated by training a person without prior experience with working on robots with a 45 minutes of training. The person could successfully perform simple tasks such as lift, rotate, insert and so on with the telerobot system. Efficiency of completion of task is evaluated based on the accuracy, steps involved and completion time. The generalized equation of this analysis is given as follows

$$
\begin{gathered}
I_{s e g}=f_{p}+4\left(n+f_{r}\right) \\
I_{M M}=f_{p}+n \\
\eta=I_{\text {seg }} / I_{M M}
\end{gathered}
$$

Where $f_{p}$ is the number of failures of planner, $f_{r}$ is the reachability failure count during segregated planning navigation and $n$ is the end-effector relocation step position. $I_{s e g}$ and $I_{M M}$ is the number of instructions in segregated and MM planning mode respectively. $\eta$ is the relation between $\mathrm{I}_{\mathrm{MM}}$ and $\mathrm{I}_{\text {seg. }}$.

The time delay in the communication between the master and slave environment is given by 


$$
T_{\text {com }}(t)=\left\{\begin{array}{c}
T_{1}(t)=200+55 \operatorname{Sin}(2 \pi t), \text { Forward } \\
T_{2}(t)=300+35 \operatorname{Sin}\left(2 \pi t+\frac{\pi}{2}\right), \text { Backward }
\end{array}\right.
$$

\section{CONCLUSION AND FUTURE SCOPE}

The field of surgery is greatly influenced by the drastic developments in information technology. Precision and time-delay have substantial impact on the performance of surgical telerobotics. This paper presents an improved system that includes supervisory control, haptic feedback and motion scaling with augmented reality. Factors like communication delay, lack of precision and signal latency are improved. The results of simulation show that the system has superior performance compared to the conventional telesurgery system. Future work involves experimental verification of formal analysis of the system. Further, addition of virtual fixtures enabling visualization of sensor data and providing a touch-screen interface is proposed as future work.

\section{References}

[1] Mylonas, George P., Ka-Wai Kwok, Ara Darzi, and Guang-Zhong Yang. "Gaze-contingent motor channelling and haptic constraints for minimally invasive robotic surgery." In International Conference on Medical Image Computing and Computer-Assisted Intervention, pp. 676-683. Springer, Berlin, Heidelberg, 2008.

[2] Shahzad, Noman, Tabish Chawla, and Tanzeela Gala. "Telesurgery prospects in delivering healthcare in remote areas." The Journal of the Pakistan Medical Association 69, no. Supl. 1 (2019): S69.

[3] Liu, Chao, Jing Guo, and Philippe Poignet. "Nonlinear Model-Mediated Teleoperation for Surgical Applications under Time Variant Communication Delay." IFAC-PapersOnLine 51, no. 22 (2018): 493-499.

[4] Hung, Andrew J., Jian Chen, Ankeet Shah, and Inderbir S. Gill. "Telementoring and telesurgery for minimally invasive procedures." The Journal of urology 199, no. 2 (2018): 355-369.

[5] Mewes, Andre, Bennet Hensen, Frank Wacker, and Christian Hansen. "Touchless interaction with software in interventional radiology and surgery: a systematic literature review." International journal of computer assisted radiology and surgery 12, no. 2 (2017): 291-305. 
Journal of Artificial Intelligence and Capsule Networks (2019)

Vol.01/ No. 01,

Pages: $28-36$

http://irojournals.com/aicn/

DOI: https://doi.org/10.36548/jaicn.2019.1.004

[6] Valner, Robert, Karl Kruusamäe, and Mitch Pryor. "TeMoto: Intuitive Multi-Range Telerobotic System with Natural Gestural and Verbal Instruction Interface." Robotics 7, no. 1 (2018): 9.

[7] Ladoiye, Jasmeet Singh, Dan S. Necsulescu, and Jurek Z. Sasiadek. "Force Control of Surgical Robot with Time Delay using Model Predictive Control." In ICINCO (2), pp. 202-210. 2018.

[8] Sheridan, Thomas B. "Space teleoperation through time delay: Review and prognosis." IEEE Transactions on robotics and Automation 9, no. 5 (1993): 592-606.

[9] Ganjefar, Soheil, Hamidreza Momeni, and Farrokh Janabi-Sharifi. "Teleoperation systems design using augmented wave-variables and Smith predictor method for reducing time-delay effects." In Proceedings of the IEEE Internatinal Symposium on Intelligent Control, pp. 333-338. IEEE, 2002.

[10] Marescaux, Jacques, Michelle K. Smith, Daniel Fölscher, Faek Jamali, Benoit Malassagne, and Joel Leroy. "Telerobotic laparoscopic cholecystectomy: initial clinical experience with 25 patients." Annals of surgery 234 , no. 1 (2001): 1 .

[11] Muszynski, Sebastian, Jörg Stückler, and Sven Behnke. "Adjustable autonomy for mobile teleoperation of personal service robots." In 2012 IEEE RO-MAN: The 21st IEEE International Symposium on Robot and Human Interactive Communication, pp. 933-940. IEEE, 2012.

[12] Stark, Michael, Emilio Ruiz Morales, and Stefano Gidaro. "Telesurgery is promising but still need proof through prospective comparative studies." Journal of gynecologic oncology 23, no. 2 (2012): 134-135.

[13] Zhou, Tian, Maria E. Cabrera, Juan P. Wachs, Thomas Low, and Chandru Sundaram. "A comparative study for telerobotic surgery using free hand gestures." Journal of Human-Robot Interaction 5, no. 2 (2016): 1-28.

[14] Khan, Arshia, and Yumna Anwar. "Robots in Healthcare: A Survey." In Science and Information Conference, pp. 280-292. Springer, Cham, 2019.

[15] Su, Hao, Weijian Shang, Gang Li, Niravkumar Patel, and Gregory S. Fischer. "An MRI-guided telesurgery system using a Fabry-Perot interferometry force sensor and a pneumatic haptic device." Annals of biomedical engineering 45, no. 8 (2017): 1917-1928. 
Journal of Artificial Intelligence and Capsule Networks (2019)

Vol.01/ No. 01,

Pages: 28-36

http://irojournals.com/aicn/

DOI: https://doi.org/10.36548/jaicn.2019.1.004

[16] Zhang, Qi, Jianhui Liu, and Guodong Zhao. "Towards 5G enabled tactile robotic telesurgery." arXiv preprint arXiv:1803.03586 (2018).

[17] Reichenbach, Mark, Tom Frederick, Lou Cubrich, Walter Bircher, Nathan Bills, Marsha Morien, Shane Farritor, and Dmitry Oleynikov. "Telesurgery with miniature robots to leverage surgical expertise in distributed expeditionary environments." Military medicine 182, no. suppl_1 (2017): 316-321.

[18] Sharifi, Mojtaba, Saeed Behzadipour, and Hassan Salarieh. "Nonlinear bilateral adaptive impedance control with applications in telesurgery and telerehabilitation." Journal of Dynamic Systems, Measurement, and Control 138, no. 11 (2016): 111010.

[19] Xu, Song, Manuela Perez, Kun Yang, Cyril Perrenot, Jacques Felblinger, and Jacques Hubert. "Effect of latency training on surgical performance in simulated robotic telesurgery procedures." The International Journal of Medical Robotics and Computer Assisted Surgery 11, no. 3 (2015): 290-295.

[20] Kolbari, Hamidreza, Soroush Sadeghnejad, Mohsen Bahrami, and Kamali E. Ali. "Adaptive Control of a Robot-Assisted Tele-Surgery in Interaction With Hybrid Tissues." Journal of Dynamic Systems, Measurement, and Control 140, no. 12 (2018): 121012.

[21] Weber, Bernhard, and Clara Eichberger. "The benefits of haptic feedback in telesurgery and other teleoperation systems: a meta-analysis." In International Conference on Universal Access in Human-Computer Interaction, pp. 394-405. Springer, Cham, 2015.

[22] Park, Sangsoo, Sang-Yun Baek, and Jeha Ryu. "Optimization for discriminability of soft tissues in haptic tele-surgery system with flexible surgical tool." In 2016 IEEE International Conference on Advanced Intelligent Mechatronics (AIM), pp. 1382-1385. IEEE, 2016.

[23] Shabana, N., and G. Velmathi. "Advanced Tele-surgery with IoT Approach." In Intelligent Embedded Systems, pp. 17-24. Springer, Singapore, 2018.

[24] Lum, Mitchell JH, Jacob Rosen, Hawkeye HI King, Diana CW Friedman, Gina Donlin, Ganesh Sankaranarayanan, Brett M. Harnett et al. "Telesurgery via unmanned aerial vehicle (UAV) with a field deployable surgical robot." In MMVR, pp. 313-315. 2007. 
Journal of Artificial Intelligence and Capsule Networks (2019)

Vol.01/ No. 01,

Pages: $28-36$

http://irojournals.com/aicn/

DOI: https://doi.org/10.36548/jaicn.2019.1.004

[25] Bar-Cohen, Yoseph, Constantinos Mavroidis, Mourad Bouzit, Benjamin P. Dolgin, Deborah L. Harm, George E. Kopchok, and Rodney A. White. "Virtual reality robotic telesurgery simulations using MEMICA haptic system." In Smart Structures and Materials 2001: Electroactive Polymer Actuators and Devices, vol. 4329, pp. 357-363. International Society for Optics and Photonics, 2001.

[26] Ottensmeyer, Mark P., Jianjuen Hu, James M. Thompson, Jie Ren, and Thomas B. Sheridan. "Investigations into performance of minimally invasive telesurgery with feedback time delays." Presence: Teleoperators \& Virtual Environments 9, no. 4 (2000): 369-382. 